\title{
Effects of host age and size on clutch size and sex ratio of Oomyzus sokolowskii (Hymenoptera: Eulophidae), a larval-pupal parasitoid of Plutella xylostella (Lepidoptera: Yponomeutidae)
}

\author{
Akiko Nakamura and Takashi Noda ${ }^{1, *}$ \\ Department of Bioscience and Technology, Faculty of Agriculture, Iwate University, Morioka 020-8550, Japan \\ ${ }^{1}$ National Institute of Agrobiological Sciences, Tsukuba, Ibaraki 305-8634, Japan \\ (Received 21 November 2001; Accepted 13 February 2002)
}

\begin{abstract}
We investigated the effects of host age and size on clutch size and sex ratio of Oomyzus sokolowskii (Kurdjumov) (Hymenoptera: Eulophidae). The number of eggs laid (primary clutch size) tended to increase with host age and was significantly larger for the late fourth-stadium larvae than for the second- and third-stadium larvae. The number of wasps that emerged from a host (secondary clutch size) tended to increase with host age and was significantly larger for the late fourth-stadium hosts than for the second-stadium hosts. In the late fourth-stadium hosts, secondary clutch size tended to increase significantly with host size. There were no significant differences in sex ratios among host ages or sizes. Where fewer than 10 wasps emerged from a host, most clutches produced only 1 male; larger clusters tended to produce 2 or more males. This implies that $O$. sokolowskii may deposit male eggs in the early eggs of a clutch and place the second male eggs in the tenth or later eggs of a clutch. By this mechanism, $O$. sokolowskii is thought to produce an invariable sex ratio regardless of clutch size.
\end{abstract}

Key words: Oomyzus (Tetranychus) sokolowskii, gregarious parasitoid, koinobiont, clutch size, sex ratio

\section{INTRODUCTION}

Host quality significantly influences the offspring fitness of parasitoid wasps (Charnov and Skinner, 1984; King, 1987; Godfray, 1994). One of the most common qualities studied is host size. It has been frequently found that host size influences the clutch size of parasitoids. Salt (1961) listed 10 studies of different parasitoid species that had shown that female wasps laid larger clutches on bigger hosts, and Godfray (1994) mentioned that a similar list now would probably contain over a hundred species. Host size also influences the parasitoid sex ratio, correlating negatively with the proportion of male progeny (Charnov et al., 1981; King, 1987, 1988, 1993; Heinz and Parrella, 1990; Ueno and Tanaka, 1997; Ueno, 1998). Host size usually increases with host age. However, host size and age may need to be considered independently because of possible physiological differences among host ages. For example, Trichogramma minutum deposited more eggs in younger hosts than in older ones of the same size, because old eggs pro- vide fewer resources for the parasitoid larvae than young eggs (Marston and Ertle, 1969). Clarifying the effects of host age and size on parasitoid reproductive characters will not only allow a better understanding of the ecological strategies of parasitoids, but it will also be useful for developing augmenting parasitoids as biological control agents.

Oomyzus (=Tetranychus) sokolowskii (Kurdjumov) (Hymenoptera: Eulophidae) is a gregarious larval-pupal endoparasitoid of the diamondback moth, Plutella xylostella (L.) (Lepidoptera: Yponomeutidae). This koinobiont parasitoid is distributed worldwide and is the only chalcidoid to have shown potential for the biological control of $P$. xylostella (Fitton and Walker, 1992). Since this parasitoid seems to be well adapted to high temperature conditions, it has been introduced into tropical and subtropical areas to control P. xylostella (Talekar and Hu, 1996). Noda et al. (1996) also reported that parasitism by $O$. sokolowskii in northern Japan was highest from August through September $(40-80 \%)$. In a previous paper (Nakamura and Noda, 2001), we demonstrated O. sokolowskii

\footnotetext{
* To whom correspondence should be addressed at: E-mail: nodat@affrc.go.jp
} 
could parasitize second- to late fouth-stadium host larvae. This implies the host size has a large variation. In this paper, we describe the results of a laboratory study carried out to investigate whether host age and size affect the clutch size and offspring's sex ratio of $O$. sokolowskii and discuss the suitability of different host age and size for parasitization.

\section{MATERIALS AND METHODS}

Insect resources and general procedures. The O. sokolowskii culture used in this study was established from parasitized host pupae collected in a cabbage field in Morioka, northern Japan, in 1997. The P. xylostella culture used for rearing the parasitoid originated from insects collected in Tsu, Mie, in 1983. Host larvae were reared on radish sprouts in a small plastic cup $(9 \mathrm{~cm}$ diameter and $5 \mathrm{~cm}$ depth) at $15^{\circ} \mathrm{C}, 16 \mathrm{~h}$ light: $8 \mathrm{~h}$ dark (LD $16: 8$ ), until parasitization. Parasitoids were reared by presenting third- and fourth-stadium larvae to a female in a glass tube $(2 \mathrm{~cm}$ diameter and $10 \mathrm{~cm}$ long). Parasitized host larvae were reared at $25^{\circ} \mathrm{C}$. Newly emerged $O$. sokolowskii females were kept in glass tubes and fed honey at $15^{\circ} \mathrm{C}$, LD 16:8 with males to ensure insemination. Only 3- to 7day-old females with no previous oviposition experience were used for the experiments.

Parasitization experiments were performed in polystyrene tubes $(1.5 \mathrm{~cm}$ diameter and $6.5 \mathrm{~cm}$ long) with radish sprouts at $25^{\circ} \mathrm{C}, \mathrm{LD} 16: 8$. One $P$. xylostella larva was presented to one female parasitoid and the oviposition behavior was observed. Immediately after the host was parasitized, the wasp was removed and the parasitized host was reared in a tube with radish sprouts at $25^{\circ} \mathrm{C}, \mathrm{LD}$ $16: 8$. Pupated hosts were singly transferred to 1.5 $\mathrm{ml}$ polypropylene microtubes and the emergence of both parasitoids and hosts was checked daily.

Effect of host age on clutch size and sex ratio of the parasitoid. Second-, third-, early fourth(within $24 \mathrm{~h}$ after molting), and late fourth-stadium (=prepupa whose cocoon web was removed) $P$. $x y$ lostella larvae were each parasitized by a single female parasitoid. Twenty parasitized larvae of each age were dissected after the completion of parasitization to count the actual number of eggs laid per host (= primary clutch size). More than 43 hosts of each age were reared individually in the polystyrene tubes with radish sprouts at $25^{\circ} \mathrm{C}, \mathrm{LD} 16: 8$ to count the number of parasitoid progeny that emerged per host (= secondary clutch size) and to calculate their sex ratios.

Effect of host size on clutch size and sex ratio of the parasitoid. Late fourth-stadium host larvae (=prepupa whose cocoon web was removed) of different sizes were each parasitized by a single female parasitoid. Only late fourth-stadium larvae were used for this experiment to avoid the influence of any differences in physiological condition of the different host ages. Parasitized host larvae were reared individually in polystyrene tubes at $25^{\circ} \mathrm{C}$, LD 16:8 to count the number of parasitoid progeny that emerged (=secondary clutch size). After the parasitoids emerged, the body length of the host pupae was measured under a binocular microscope. Because the hosts used in this experiment were prepupae that were no longer feeding, we consider that their size represented the body size when they were parasitized.

\section{RESULTS}

\section{Effect of host age on clutch size and sex ratio of the parasitoid}

Primary clutch size tended to increase with host age except in third-stadium larvae and was significantly larger for the late fourth-stadium larvae than for the second- and third-stadium larvae (Table 1). The number of wasps that emerged from a host (=secondary clutch size) also tended to increase with host age, and was significantly larger for the late fourth-stadium hosts than for the secondstadium hosts. There were no significant differences in sex ratio among host sizes.

\section{Effect of host size on clutch size and sex ratio of the parasitoid}

The number of progeny that emerged per host (=secondary clutch size) increased significantly with host size (regression analysis; $F=4.421$, $p=0.0396$; Fig. 1a). The primary clutch sizes were not measured. There were no significant differences in sex ratio among host sizes (regression analysis; $F=0.225, p=0.6372$; Fig. $1 \mathrm{~b}$ ). The smallest clutch size was 3 and the largest was 20. Figure 2 shows the number of males that emerged from a clutch in relation to the secondary clutch size. When the clutch size was less than 10, most clutches produced only 1 male. When the clutch 
Table 1. Effect of host age on primary clutch size, secondary clutch size, and offspring sex ratio

\begin{tabular}{lccc}
\hline $\begin{array}{c}\text { Host age } \\
\text { (stadium) }\end{array}$ & $\begin{array}{c}\text { Primary } \\
\text { clutch size }^{\mathrm{a}}\end{array}$ & $\begin{array}{c}\text { No. wasps } \\
\text { emerged }^{\text {per host }}{ }^{\mathrm{a}}\end{array}$ & $\begin{array}{c}\text { Sex ratio } \\
(\% \text { males })^{\mathrm{b}}\end{array}$ \\
\hline 2nd & $\begin{array}{c}11.4 \pm 0.8 \mathrm{a} \\
(20)\end{array}$ & $\begin{array}{c}9.2 \pm 0.3 \mathrm{a} \\
(63)\end{array}$ & 10.7 \\
3rd & $\begin{array}{c}10.2 \pm 1.5 \mathrm{a} \\
(20)\end{array}$ & $\begin{array}{c}10.1 \pm 0.4 \mathrm{ab} \\
(60)\end{array}$ & 12.4 \\
Early 4th & $\begin{array}{c}12.2 \pm 0.3 \mathrm{ab} \\
(20)\end{array}$ & $\begin{array}{c}9.6 \pm 0.5 \mathrm{ab} \\
(43)\end{array}$ & 13.2 \\
Late 4th & $\begin{array}{c}16.6 \pm 1.6 \mathrm{~b} \\
(20)\end{array}$ & $\begin{array}{c}11.1 \pm 0.6 \mathrm{~b} \\
(47)\end{array}$ & 11.7 \\
& & & \\
\hline
\end{tabular}

${ }^{\mathrm{a}}$ Mean \pm SE. Means followed by the same letter do not differ significantly at $p=0.05$ by Tukey-Kramer's test. Numbers of hosts used are shown in parentheses.

${ }^{\mathrm{b}}$ No significant differences were found in the sex ratio at $p=$ 0.05 by $\mathrm{R} \times \mathrm{C}$ tests of independence using chi-square test.
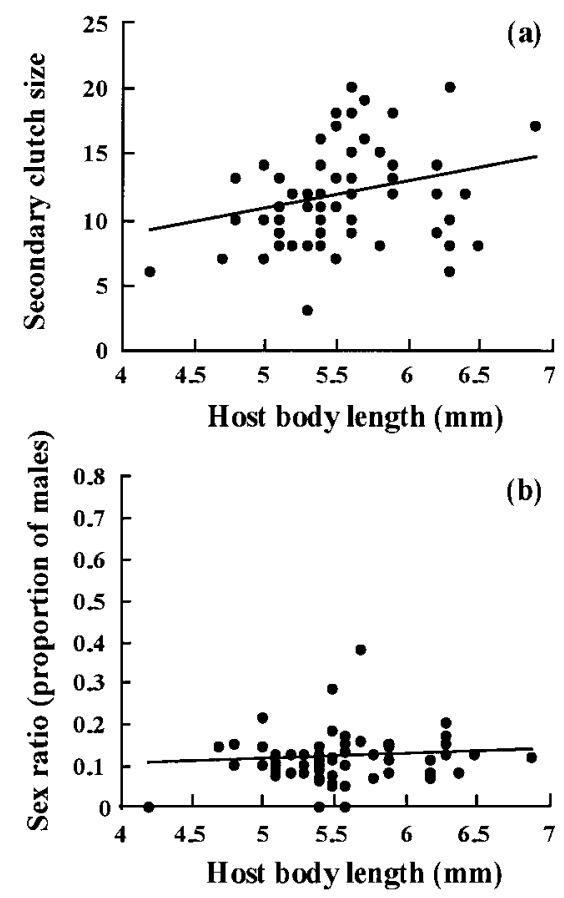

Fig. 1. (a) Relationship between host size and secondary clutch size $\left(y=2.043 x+0.595 ; R^{2}=0.067 ; x=\right.$ host body length in $\mathrm{mm}$ ). (b) Relationship between host size and sex ratio $\left(y=0.012 x+0.059 ; R^{2}=0.004 ; x=\right.$ host body length in $\left.\mathrm{mm}\right)$.

size was 10 or more, clutches often produced 2 or more males.

\section{DISCUSSION}

The primary clutch sizes tended to increase with host age (Table 1). Such a positive correlation be-

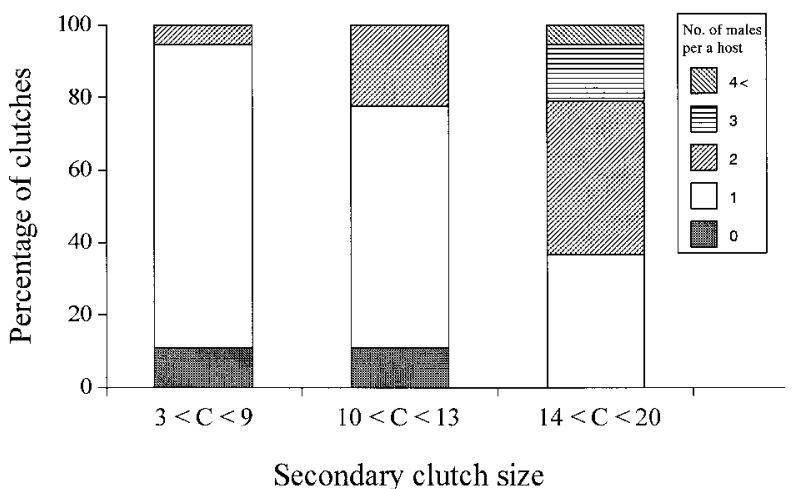

Fig. 2. Secondary clutch size (C) and number of males emerged from a clutch. Numbers of clutches involved in each clutch size ranges are: 18 in " $3<\mathrm{C}<9$ ", 27 in " $10<\mathrm{C}<13$," and 19 in " $14<\mathrm{C}<20$," respectively.

tween host age and parasitoid clutch size has been reported for many parasitoid wasps (Salt, 1941; Klomp and Teerink, 1962). This suggests that a parasitoid can recognize the host size and adjust the clutch size accordingly. In our previous paper (Nakamura and Noda, 2001), we demonstrated that the duration of ovipositor insertion and oviposition of $O$. sokolowskii was significantly longer on fourth-stadium hosts than on second- and thirdstadium hosts. We also demonstrated that the time taken for drumming by antennae was slightly longer on fourth-stadium hosts than on other stadia, although not significant. These behavioral differences may indicate a difference in chemical and/or visual conditions between small and large hosts and explain how the parasitoid recognizes host size. The secondary clutch sizes were significantly smaller than the primary ones in all host stadia except for the third-stadium ( $t$-test, $p<0.05$ ), probably because of larval death. However, the secondary clutch sizes in the fourth-stadium hosts were also larger than those in the other stadia. Wang et al. (1999) reported that the number of $O$. sokolowskii progeny per host pupa did not differ significantly among host ages. They put 5 parasitoids and 24 hosts together in a container for 24 h. Such a condition can cause several females to oviposit into the same host and consequently equalize the number of progeny per host. In our study, we presented a single host to a single parasitoid under direct observation. This difference in methods may explain the difference in results.

Host age and host size are usually related to growing stage. However, host quality may be influ- 
enced by the internal physiological condition of the host, such as hormonal balance. Host size and age are mutually confounding factors when larval hosts are used. Therefore, we used late fourth-stadium (=prepupa) hosts of different sizes to test the effects of host size on parasitoid reproduction. Consequently, the secondary clutch size tended to increase significantly with host size (Fig. 1a) as well as with host age. Host size has been frequently found to correlate negatively with the proportion of male progeny (Charnov et al., 1981; King, 1987, 1988, 1993; Heinz and Parrella, 1990; Ueno and Tanaka, 1997; Ueno, 1998). However, we found no significant differences in sex ratio among host sizes. This result suggests that the female wasps can place more male eggs in larger hosts in response to the host size and adjust the offspring sex ratio. In fact, the relationship between the secondary clutch size and the number of males per clutch showed that the female produced only 1 male when the clutch size was 9 or fewer and 2 or more when the clutch size was 10 or more (Fig. 2). This implies that $O$. sokolowskii may be able to optimize the sex ratio, as some trichogrammatids and scelionids do (Waage, 1982; Waage and $\mathrm{Ng}, 1982$; Noda and Hirose, 1989). Such a sex-ratio control has been found for many parasitoid species in the LMC type situation (Godfray, 1994) where a few males can inseminate many of their sisters. Because $O$. sokolowskii shows habitually inbreeding or sibmating, it is considered that the sex ratio of O. sokolowskii can be influenced by host size under LMC situations.

In our previous paper (Nakamura and Noda, 2001), we found that fourth-stadium larvae were more suitable hosts for $O$. sokolowskii than secondstadium larvae, because they produced higher successful parasitism and lower parasite mortality. Our latest results - that clutch size increased with host size-support this conclusion.

\section{REFERENCES}

Charnov, E. L., R. L. Ros-den Hartogh, W. T. Jones and J. van den Assem (1981) Sex ratio evolution in a variable environment. Nature 289: 27-33.

Charnov, E. L. and S. W. Skinner (1984) Evolution of host selection and clutch size in parasitoid wasps. Florida Entomol. 67: 5-21.

Fitton, M. and A. Walker (1992) Hymenopterous parasitoids associated with diamondback moth: the taxonomic dilemma. In Diamondback Moth and Other Crucifer Pests: Proceedings the Second International Workshop
(N. S. Talekar ed.). Asian Vegetable Research and Development Center, Taipei, pp. 225-232.

Godfray, H. C. J. (1994) Parasitoids: Behavioral and Evolutionary Ecology. Princeton Univ. Press, New Jersey. 473 pp.

Heinz, K. M. and M. P. Parrella (1990) The influence of host size on sex ratios in the parasitoid Diglyphus begini (Hymenoptera: Eulophidae). Ecol. Entomol. 15: 391-399.

King, B. H. (1987) Offspring sex ratios in parasitoid wasps. $Q$. Rev. Biol. 62: 367-396.

King, B. H. (1988) Sex-ratio manipulation in response to host size by the parasitoid wasp Splangia cameroni: a laboratory study. Evolution 42: 1190-1198.

King, B. H. (1993) Sex ratio manipulation by parasitoid wasps. In Evolution and Diversity of Sex Ratio in Insects and Mites (D. L. Wrensch and M. A. Ebbert eds.). Chapman and Hall, New York, pp. 418-441.

Klomp, H. and B. J. Teerink (1962) Host selection and number of eggs per oviposition in the egg parasite Trichogramma embryophagum Htg. Nature 195: 1020-1021.

Marston, N. and L. R. Ertle (1969) Host age and parasitism by Trichogramma minutum (Hymenoptera: Trichogrammatidae). Ann. Entomol. Soc. Am. 62: 1476-1482.

Nakamura, A. and T. Noda (2001) Host-age effects on oviposition behavior and development of Oomyzus sokolowskii (Hymenoptera: Eulophidae), a larval-pupal parasitoid of Plutella xylostella (Lepidoptera: Yponomeutidae). Appl. Entomol. Zool. 36: 367-372.

Noda, T. and Y. Hirose (1989) "Males second" strategy in the allocation of sexes by the parasitic wasp, Gryon japanicum. Oecologia 81: 145-148.

Noda, T., S. Miyai, S. Yamada and K. Konishi (1996) Larval and pupal parasitoids of diamondback moth, Plutella $x y$ lostella (L.) in cabbage fields in Morioka, Japan. Jpn. J. Appl. Entomol. Zool. 40:164-167 (in Japanese with English summary).

Salt, G. (1941) The effects of hosts upon their insect parasites. Biol. Rev. 16: 239-264.

Salt, G. (1961) Competition among insect parasitoids. Mechanisms in biological competition. Symp. Soc. Exp. Biol. 15: 96-119.

Talekar, N. S. and W. J. Hu (1996) Characteristics of parasitism of Plutella xylostella (Lep., Plutellidae) by Oomyzus sokolowskii (Hym., Eulophidae). Entomophaga 41: 4552.

Ueno, T. (1998) Sex allocation by a parasitoid wasp (Hymenoptera: Ichneumonidae) to different host species: a question for the mechanism of host size estimation. J. Insect Behav. 11: 811-821.

Ueno, T. and T. Tanaka (1997) Comparison between primary and secondary sex ratios in parasitoid wasps using a method for observing chromosomes. Entomol. Exp. Appl. 82: 105-108.

Waage, J. K. (1982) Sib-mating and sex ratio strategies in scelionid wasps. Ecol. Entomol. 7: 103-112.

Waage, J. K. and S. M. Ng (1982) The reproductive strategy of a parasitic wasp. I. Optimal progeny allocation in Trichogramma evanescens. J. Anim. Ecol. 53: 401-415.

Wang, X., S. Liu, S. Guo and W. Lin (1999) Effects of host stages and temperature on population parameters of Oomyzus sokolowskii, a larval-pupal parasitoid of Plutella xylostella. Biocontrol 44: 391-402. 